Dieses Dokument ist eine Zweitveröffentlichung (Verlagsversion) /

This is a self-archiving document (published version)

„Dieser Beitrag ist mit Zustimmung des Rechteinhabers aufgrund einer (DFG-geförderten) Allianzbzw. Nationallizenz frei zugänglich."

This publication is openly accessible with the permission of the copyright owner. The permission is granted within a nationwide license, supported by the German Research Foundation (abbr. in German DFG).

https://www.nationallizenzen.de
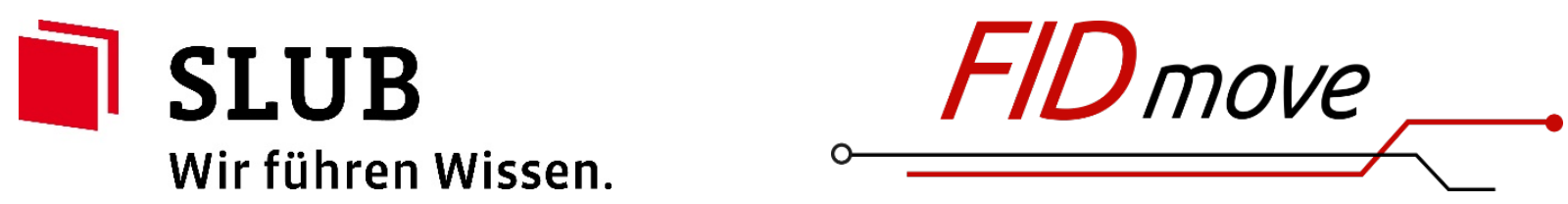


\title{
Stress and failure analysis of thick-walled conical composite rotors
}

W Hufenbach, M Gude*, B Zhou and L Kroll

Institut für Leichtbau und Kunststofftechnik (ILK), Technische Universität Dresden, Dresden, Germany

\begin{abstract}
The high specific strength and stiffness of composite materials, as well as the possibility of creating a load-adapted property profile of them are ideally suited for the design of high-speed lightweight rotors. With respect to a load-adapted reinforcement structure of composite rotors, the rotor geometry has a significant influence on the optimum fibre orientation. In the case of conical rotors-as opposed to discshaped and cylindrical rotors - the structural behaviour is strongly influenced by centrifugally induced bending effects in the rotor structure, which cause complex three-dimensional stress states in combination with the ordinary tangential and radial stresses. For analysis of the resulting complex stress states, an analytical method has been developed and verified numerically as well as experimentally. The novel method presented here is the basis for a realistic failure analysis and, in particular, serves as an efficient tool for extensive parameter studies and optimizations within the design process.
\end{abstract}

Keywords: composite rotors, conical rotors, three-dimensional stress analysis, failure analysis, spin testing

NOTATION
$C_{i j}$
$L$
$L_{j m}$
$M_{s}, M_{\varphi}, M_{t}$
$N$
$p_{\perp \perp}, p_{\perp \|}$
$P_{i}$
$r_{0}$
$R_{\perp \perp}$
$R_{\perp}$
$R_{\perp \perp}^{\mathrm{A}}$
$R_{\|}^{( \pm)}$
$R_{\perp}^{(+)}$
$s, \varphi, t$
$s^{*}, t^{\bullet}$
$u_{s}, u_{\varphi}, u_{t}$
$U_{m}^{i p}, V_{m}^{i p}, W_{m}^{i p}$
$\bar{U}$
$V$

stiffness tensor

cone shell length in the meridional direction corresponding beam solutions order of the Ritz approach number of unidirectional layers proportionality factors order of the Bernstein function maximum cone diameter transverse/transverse shear strength transverse/parallel shear strength action plane resistance tensile/compressive strength in the fibre direction tensile strength perpendicular to fibres cylindrical coordinate system normalized coordinates displacement in the $s, \varphi$ and $t$ directions Ritz coefficients strain energy density volume

The MS was received on 20 March 2003 and was accepted after revision for publication on 16 October 2003.

*Corresponding author: Institut für Leichtbau und Kunststofftechnik, Technische Universität Dresden, 012062 Dresden, Germany.

$\begin{array}{ll}X_{j} & \text { body forces } \\ \alpha & \text { cone angle } \\ \alpha_{s m}, \alpha_{t m}, \beta_{s m}, \beta_{t m} & \begin{array}{l}\text { eigenvalues of transcendental } \\ \text { eigenvalue equations }\end{array} \\ \gamma_{\varphi t}, \gamma_{t s}, \gamma_{s \varphi} & \text { shear strains } \\ \varepsilon_{s}, \varepsilon_{\varphi}, \varepsilon_{t} & \text { strains in the } s, \varphi \text { and } t \text { directions } \\ \Pi & \text { total potential of the elastic body } \\ \rho_{i} & \text { density of the } i \text { th unidirectional layer } \\ \sigma_{\mathrm{n}}, \tau_{\mathrm{n} 1}, \tau_{\mathrm{nt}} & \text { fracture plane related stresses } \\ \sigma_{s}, \sigma_{\varphi}, \sigma_{t} & \text { normal stresses in an orthogonal } \\ & \text { curvilinear coordinate system } \\ \sigma_{1}, \sigma_{2}, \sigma_{3} & \text { normal stresses in a fibre-adapted } \\ \tau_{\varphi t}, \tau_{t s}, \tau_{s \varphi} & \text { coordinate system } \\ & \text { shear stresses in a cylindrical } \\ \tau_{21}, \tau_{23}, \tau_{31} & \text { coordinates system } \\ & \text { shear stresses in a fibre-adapted } \\ \phi_{\mathrm{p}}^{P_{i}} & \text { coordinate system } \\ \omega & \text { Bernstein polynomial } \\ & \text { angular velocity }\end{array}$

\section{INTRODUCTION}

Advanced high-speed rotors, which are subjected to extreme centrifugal forces, can efficiently be realized using long fibre reinforced composite materials. In particular, anisotropic polymer composites, with their excellent lightweight characteristics, corrosion resistance and wide scope for designability - such as the possibility of adjusting the strength,

Proc. Instn Mech. Engrs Vol. 218 Part L: J. Materials: Design and Applications 

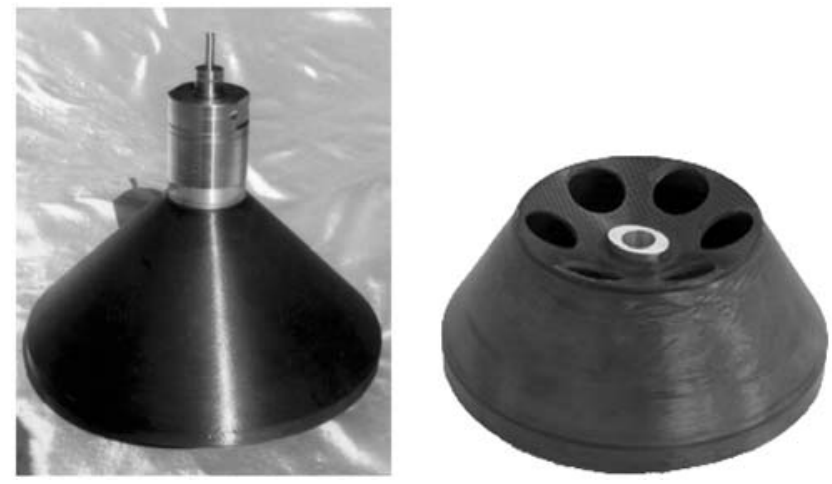

Fig. 1 Conical composite rotor $\left(\varnothing_{\mathrm{a}}=435 \mathrm{~mm}\right)$ and a composite centrifuge rotor $\left(\varnothing_{\mathrm{a}}=200 \mathrm{~mm}\right)$ [2]

stiffness and thermal expansion behaviour-offer a new range of performance in process technology e.g. for decanting centrifuges, air classifiers or turbines [1].

For optimum utilization of the excellent lightweight potential of fibre reinforced polymers with respect to the load-adapted design of high-speed rotors, a detailed knowledge of the mechanical behaviour of the multilayered rotor structure is essential. The description of this structural behaviour, however, is made more difficult by different effects of both the anisotropy and the rotor geometry. In particular, in the case of rotationally symmetrical but noncylindrical rotors - such as conical rotors (Fig. 1) additional bending effects due to centrifugal forces are superimposed on the radial and tangential stresses known from cylindrical and disc-shaped rotors. Consequently, the structural behaviour of non-cylindrical rotors is characterized by a three-dimensional stress state.

For realistic analysis of the arising complex stress and deformation fields of thick-walled multilayered conical rotors under centrifugal force, extended analytical calculation methods based on a three-dimensional theory have been developed and verified numerically by adapted finite element models as well as experimentally by high-speed rotor tests.

In order to assess the arising three-dimensional stress fields with respect to failure, a realistic fracture analysis of the composite structure has been carried out using the physically based Hashin/Puck failure criterion.

\section{THREE-DIMENSIONAL ANALYSIS OF A CONICAL COMPOSITE ROTOR}

Calculation of the three-dimensional stress states of the conical multilayered composite rotor under centrifugal forces is based on the principle of the minimum total potential energy in combination with the Ritz method. Therefore, linear elastic material behaviour, small deformations and a rotationally symmetrical stress state are assumed, and three-dimensional displacement approaches are chosen.

For the description of the structural behaviour of the conical rotor, an orthogonal curvilinear coordinate system $(s, \varphi, t)$ is introduced (Fig. 2). According to this coordinate

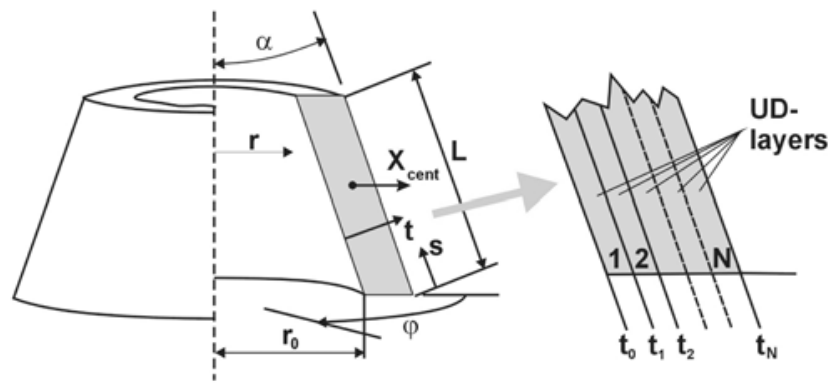

Fig. 2 Sketch of a conical composite rotor under centrifugal forces

system, the displacements $u_{s}, u_{\varphi}$ and $u_{t}$, the strains $\varepsilon_{s}, \varepsilon_{\varphi}, \varepsilon_{t}$, $\gamma_{\varphi t}, \gamma_{t s}$ and $\gamma_{s \varphi}$ as well as the stresses $\sigma_{s}, \sigma_{\varphi}, \sigma_{t}, \tau_{\varphi t}, \tau_{t s}$ and $\tau_{s \varphi}$ are defined.

The strain-displacement relations are given by

$$
\begin{aligned}
\varepsilon_{s} & =\frac{\partial u_{s}}{\partial s} \\
\varepsilon_{\varphi} & =\frac{1}{r} \frac{\partial u_{\varphi}}{\partial \varphi}+\frac{1}{r} u_{t}-\frac{\sin \alpha}{r} u_{s} \\
\varepsilon_{t} & =\cos \alpha \frac{\partial u_{t}}{\partial t} \\
\gamma_{\varphi t} & =\cos \alpha \frac{\partial u_{\varphi}}{\partial t}+\frac{1}{r} \frac{\partial u_{t}}{\partial \varphi}-\frac{1}{r} u_{\varphi} \\
\gamma_{t s} & =\frac{\partial u_{t}}{\partial s}+\cos \alpha \frac{\partial u_{s}}{\partial t} \\
\gamma_{s \varphi} & =\frac{1}{r} \frac{\partial u_{s}}{\partial \varphi}+\frac{\partial u_{\varphi}}{\partial s}+\frac{\sin \alpha}{r} u_{\varphi}
\end{aligned}
$$

with

$$
r=r_{0}-s \sin \alpha+\frac{t}{\cos \alpha}
$$

Hooke's law relating stresses to strains for an monoclinic anisotropic material can be written as

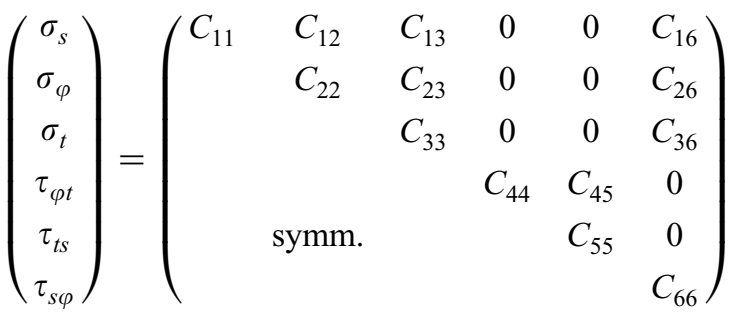

$$
\begin{aligned}
& \times\left(\begin{array}{c}
\varepsilon_{s} \\
\varepsilon_{\varphi} \\
\varepsilon_{t} \\
\gamma_{\varphi t} \\
\gamma_{t s} \\
\gamma_{s \varphi}
\end{array}\right)
\end{aligned}
$$


where $\sigma_{i}$ and $\tau_{i j}$ are the stress components, $\varepsilon_{j}$ and $\gamma_{i j}$ are the strain components and $C_{i j}$ is the stiffness matrix.

The energy principle for a rotor under centrifugal forces is given in the form

$$
\delta \Pi=\delta\left[\iiint_{V} \bar{U} \mathrm{~d} V-\iiint_{V} X_{j} u_{j} \mathrm{~d} V\right]=0
$$

with the strain energy density

$$
\begin{aligned}
\bar{U}= & \frac{1}{2} C_{11} \varepsilon_{s}^{2}+\varepsilon_{s}\left(C_{12} \varepsilon_{\varphi}+C_{13} \varepsilon_{t}+C_{16} \gamma_{s \varphi}\right) \\
& +\frac{1}{2} C_{22} \varepsilon_{\varphi}^{2}+\varepsilon_{\varphi}\left(C_{23} \varepsilon_{t}+C_{26} \gamma_{s \varphi}\right) \\
& +\frac{1}{2} C_{33} \varepsilon_{t}^{2}+C_{36} \varepsilon_{t} \gamma_{s \varphi}+\frac{1}{2} C_{44} \gamma_{\varphi t}^{2} \\
& +C_{45} \gamma_{\varphi t} \gamma_{t s}+\frac{1}{2} C_{55} \gamma_{t s}^{2}+\frac{1}{2} C_{66} \gamma_{s \varphi}^{2}
\end{aligned}
$$

and the body forces $X_{s}, X_{\varphi}$ and $X_{t}$ in the $s, \varphi$ and $t$ directions.

For application of the Ritz method, the displacement functions for a rotor of $N$ layers are given by the functions $\phi_{p}^{P_{i}}\left(t^{*}\right)$ and $L_{j m}\left(s^{*}\right)(j=s, \varphi, t)$, which are dependent on the meridian direction and thickness direction respectively

$$
\begin{aligned}
& u_{s}=\sum_{i=1}^{N} \sum_{m=1}^{M_{s}} \sum_{p=0}^{P_{i}} U_{m}^{i p} \phi_{\mathrm{p}}^{P_{i}}\left(t^{*}\right) L_{s m}\left(s^{*}\right) \\
& u_{\varphi}=\sum_{i=1}^{N} \sum_{m=1}^{M_{\varphi}} \sum_{p=0}^{P_{i}} V_{m}^{i p} \phi_{\mathrm{p}}^{P_{i}}\left(t^{\bullet}\right) L_{\varphi m}\left(s^{*}\right) \\
& u_{t}=\sum_{i=1}^{N} \sum_{m=1}^{M_{t}} \sum_{p=0}^{P_{i}} W_{m}^{i p} \phi_{\mathrm{p}}^{P_{i}}\left(t^{*}\right) L_{t m}\left(s^{*}\right)
\end{aligned}
$$

with the normalized coordinates $s^{*}=s / L$, the order of the Ritz approaches $M_{s}, M_{\varphi}, M_{t}$ and

$$
\begin{aligned}
& t^{*}=\frac{t-t_{i-1}}{\Delta t_{i}} \\
& \Delta t_{i}=t_{i}-t_{i-1}
\end{aligned}
$$

The displacement approaches (6) with the unknown coefficients $U_{m}^{i p}, V_{m}^{i p}$ and $W_{m}^{i p}$ are chosen such that the boundary conditions regarding the displacement at both cone ends, which are free at the bottom end and fixed in the $s$ and $\varphi$ directions at the head, are fulfilled. The rotor is loaded by centrifugal forces, where a free deformation in all directions is allowed. In order to forbid a rigid body motion, the functions $L_{s m}\left(s^{*}\right), L_{\varphi m}\left(s^{*}\right)$ and $L_{t m}\left(s^{*}\right)$ are introduced

$$
\begin{aligned}
L_{s m}= & \sin \left(\beta_{s m} s^{*}\right) \\
L_{\varphi m}= & \sin \left(\beta_{\varphi m} s^{*}\right) \\
L_{t m}= & \cosh \left(\beta_{t m} s^{*}\right)-\alpha_{t m} \sinh \left(\beta_{t m} s^{*}\right) \\
& \quad+\cos \left(\beta_{t m} s^{*}\right)-\alpha_{t m} \sin \left(\beta_{t m} s^{*}\right)
\end{aligned}
$$

with

$$
\begin{aligned}
\beta_{s m} & =\beta_{\varphi m}=\frac{2 m-1}{2} \pi \\
\alpha_{t m} & =\frac{\cosh \beta_{t m}-\cos \beta_{t m}}{\sinh \beta_{t m}-\sin \beta_{t m}}
\end{aligned}
$$

where $\beta_{t m}$ can be calculated as

$$
\cosh \beta_{t m} \cos \beta_{t m}-1=0
$$

The displacements have to be continuous in the thickness direction $t$, whereas their first derivative with regard to $t$ must be discontinuous in order to ensure continuous stresses in the $t$ direction. Therefore, the deficient piecewise polynomial function - the one-dimensional Bernstein function-is introduced [2]

$$
\begin{aligned}
\phi_{\mathrm{p}}^{P_{i}}\left(t^{*}\right)= \begin{cases}\left(\frac{P_{i} !}{p !\left(P_{i}-p\right) !}\right) t^{* p}\left(1-t^{*}\right)^{P_{i}-p} & \text { for } t \in\left(t_{i-1}, t_{i}\right] \\
0 & \text { otherwise }\end{cases} \\
\quad i=1,2, \ldots, N
\end{aligned}
$$

with the degrees of freedom $P_{i}$ and

$$
t^{\cdot}= \begin{cases}\frac{t-t_{i-1}}{t_{i}-t_{i-1}} & \text { for } t \in\left(t_{i-1}, t_{i}\right] \\ 0 & \text { otherwise }\end{cases}
$$

In order to enforce continuity for the displacement functions at the layer borders, the following transition conditions have to be fulfilled

$$
\begin{aligned}
& U_{m}^{i P_{i}}=U_{m}^{(i+1) 0}, \quad i=1,2, \ldots, N-1 \\
& V_{m}^{i P_{i}}=V_{m}^{(i+1) 0}, \quad i=1,2, \ldots, N-1 \\
& W_{m}^{i P_{i}}=W_{m}^{(i+1) 0}, \quad i=1,2, \ldots, N-1
\end{aligned}
$$

The centrifugal forces of the $i$ th layer of the cone are

$$
\begin{aligned}
X_{s}^{i} & =-\rho_{i} \omega^{2} r \sin \alpha, \quad i=1,2, \ldots, N \\
X_{\varphi}^{i} & =0, \quad i=1,2, \ldots, N \\
X_{t}^{i} & =\rho_{i} \omega^{2} r \cos \alpha, \quad i=1,2, \ldots, N
\end{aligned}
$$

where $\omega$ is the angular velocity and $\rho_{i}$ is the material density in the $i$ th layer.

Substitution of equations (6) and (14) into equation (4) results in a system of linear equations with

$$
\left(M_{s}+M_{\varphi}+M_{t}\right)\left[1+\sum_{i=1}^{N} P_{i}\right]
$$

equations to calculate the Ritz coefficients $U_{m}^{i p}, V_{m}^{i p}$ and $W_{m}^{i p}$ of the displacement approaches, which then serve for the

Proc. Instn Mech. Engrs Vol. 218 Part L: J. Materials: Design and Applications 
calculation of the strains and stresses by equations (1) and (3).

\section{STRUCTURAL ANALYSIS}

\subsection{Analytical stress analysis}

As an example, the mechanical behaviour of a thick-walled conical composite rotor model $\left(\alpha=22.5^{\circ}, L=176.4 \mathrm{~mm}\right.$, $r_{0}=26.3 \mathrm{~mm}$ ) with a media load has been investigated using the developed analytical model. The rotor model consists of a tangentially wound carbon fibre reinforced polymer (CFRP) layer of $10 \mathrm{~mm}$ thickness and a service load idealized as a medium layer of $35 \mathrm{~mm}$ thickness. The related material properties are given in Table 1.

The in-plane stresses in the $\varphi$ and $s$ directions with regard to the rotor thickness and length coordinates are shown in Figs 3 and 4 . The tangential stresses $\sigma_{\varphi}$ are strongly dependent on the $s$ coordinate and reach a maximum at the bottom of the inner CFRP layer (Fig. 3). By contrast, only a slight influence of the $t$ coordinate has been observed.

The stresses $\sigma_{s}$, which are caused on the one hand by bending effects and on the other hand by normal tensile loads, show a linear distribution along the rotor thickness (Fig. 4). As expected, the maximum value of $\sigma_{s}$ occurs at the top of the cone on account of the fixed support.

\subsection{Numerical structural analysis}

Besides the analytical calculations, further detailed numerical stress and deformation analyses of real conical composite rotors with different cone angles have been carried out before experimental testing. Here, the main focus has been the centrifugally induced deformation behaviour and stress states of tangentially wound CFRP rotors dependent on different cone angles $\alpha=30,45$ and $60^{\circ}$. The model has been meshed in I-DEAS with axisymmetric elements, where the inner vertical boundary has been fixed in the vertical direction. The calculated maximum radial displacements due to a load of $60000 \mathrm{r} / \mathrm{min}$ for the $45^{\circ}$ cone rotor are shown in Fig. 5 together with the reference geometry.

The resulting distribution of stresses $\sigma_{s}$ of the cone are shown in Fig. 6. Maximum compressive stresses occur on

Table 1 Material properties

\begin{tabular}{lc}
\hline CFRP & 142000 \\
$E_{\varphi}(\mathrm{MPa})$ & 9700 \\
$E_{t}, E_{s}(\mathrm{MPa})$ & 0.29 \\
$v_{\psi t}, v_{\psi s}$ & 0.31 \\
$v_{s t}$ & 3900 \\
$G_{\psi t}, G_{\psi s}(\mathrm{MPa})$ & 3240 \\
$G_{s t}(\mathrm{MPa})$ & 1600 \\
$\rho\left(\mathrm{kg} / \mathrm{m}^{3}\right)$ & \\
$\mathrm{Media}$ & 10 \\
$E(\mathrm{MPa})$ & 0.3 \\
$v$ & 1390 \\
$\rho\left(\mathrm{kg} / \mathrm{m}^{3}\right)$ & \\
\hline
\end{tabular}

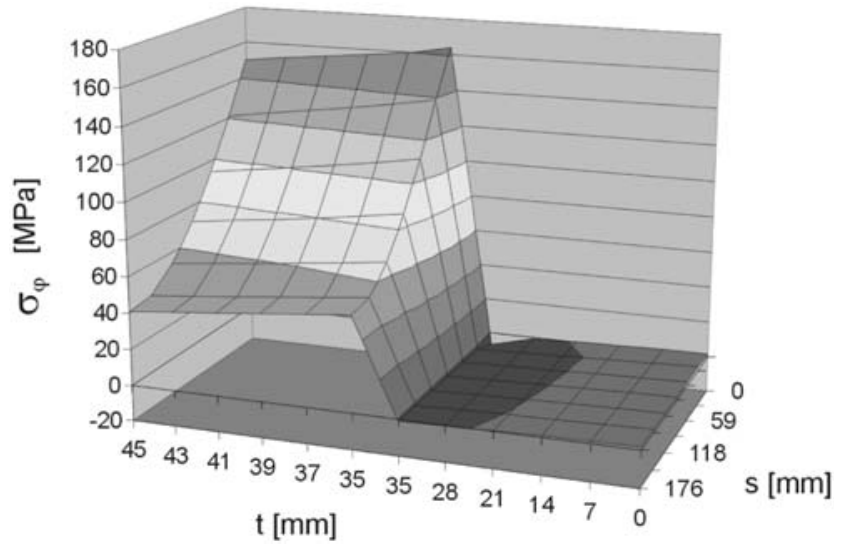

Fig. 3 Analytically calculated tangential stresses $\sigma_{\varphi}$

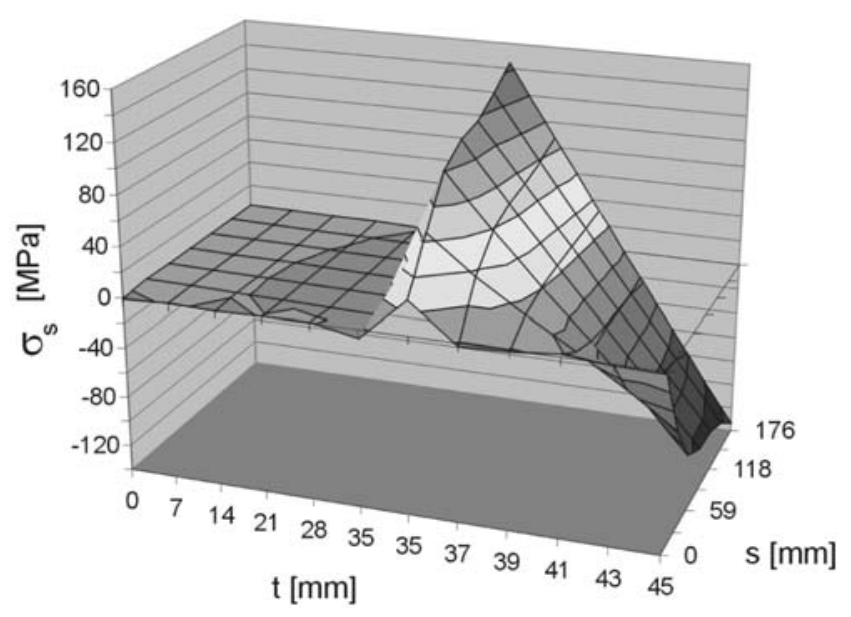

Fig. 4 Analytically calculated stresses $\sigma_{s}$

the outer cone surface, whereas maximum tensile stresses occur at the inner cone surface, which are most critical with respect to failure here since they are acting perpendicular to the fibres. For assessment of the calculated three-dimensional stress states, a realistic failure analysis

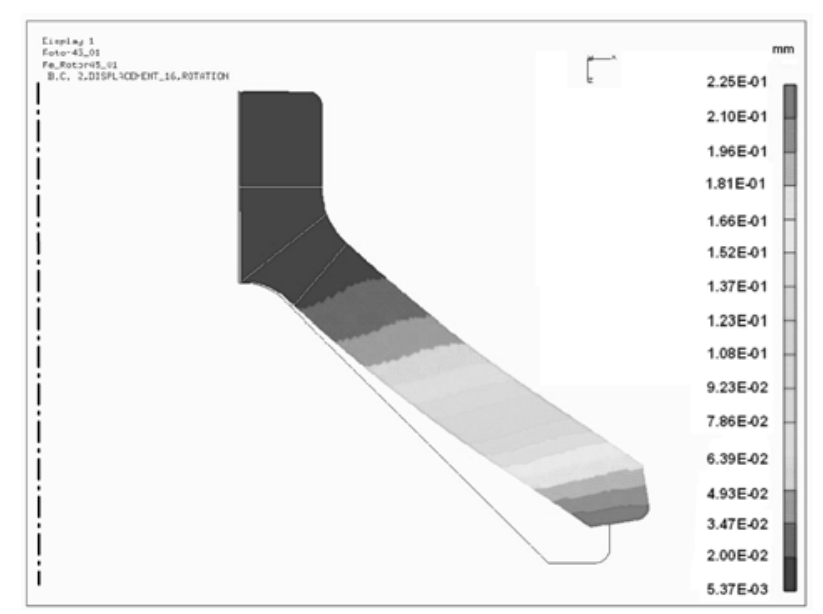

Fig. 5 Radial displacement of a conical rotor $\left(\alpha=45^{\circ}\right)$ 


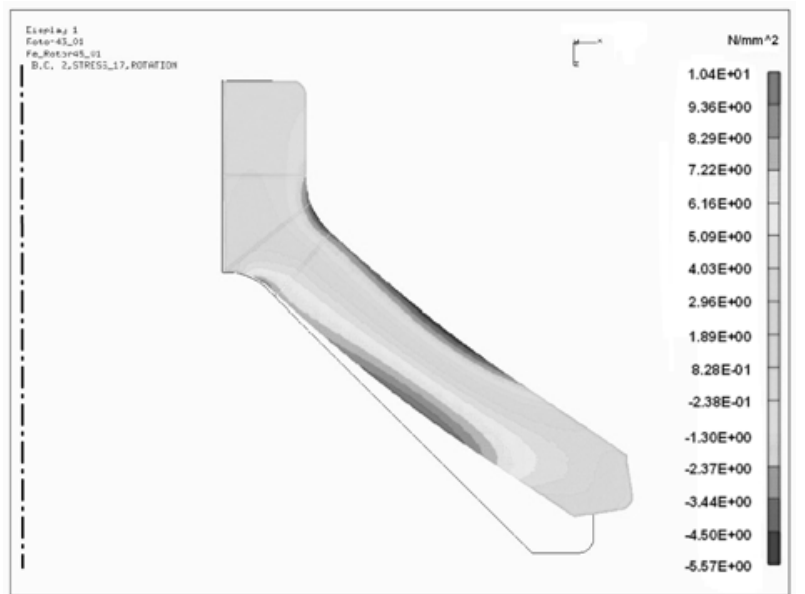

Fig. 6 Centrifugally induced bending stresses $\sigma_{s}$

of non-cylindrical composite rotors is of particular importance.

\section{FAILURE ANALYSIS}

\subsection{Physically based failure criterion}

In practice, different rotor failure mechanisms such as delamination, multifragmentation, material loss and dynamic response on initial failure have been observed. These mechanisms are dependent on the material, reinforcement structure, rotor shape and loading conditions of the composite rotor $[\mathbf{3}, \mathbf{4}]$.

For theoretical determination of the fracture stresses of thick-walled multilayered composite rotors with complex three-dimensional stress states, the conventional fracture criteria are unsuitable because these criteria do not take into account three-dimensional failure effects such as fracture modes or angles of fracture [5].

A completely different method for the formulation of realistic three-dimensional fracture criteria, taking into consideration the heterogeneous material structure relevant to the fracture, has been developed by Hashin [6] and Puck [7]. Here, not only the decisive difference between fibre fracture and interfibre fracture is considered, but a fracture angle has been introduced as a free parameter, which also characterizes further fracture types in the plane parallel to the fibres. In accordance with mechanical material behaviour and phenomenological observations, it is also taken into consideration that compressive stresses perpendicular to the fibres tend to prevent the formation of fracture, while corresponding tensile stresses tend to promote composite fracture. Thus, the new fracture criteria developed by Hashin and Puck, in contrast to the generalizing fracture criteria, are based on physical phenomena. The first approach in this direction came from Mohr for isotropic materials and has proven to be very satisfactorily in its description of brittle fracture behaviour.

Puck distinguishes two fundamental failure modes, which require two independent failure criteria: fibre failure (FF)

and interfibre failure (IFF). A simple failure criterion for fibre fracture is

$$
\left(\frac{\sigma_{1}}{R_{\|}^{( \pm)}}\right)^{2}=1
$$

where $R_{\|}^{(+)}$is the longitudinal tensile strength for $\sigma_{1}>0$, and $R_{\|}^{(-)}$is the longitudinal compression strength for $\sigma_{1}<0$. This relation describes clear fibre failure in a fixed plane perpendicular to the fibres, which is only seldom observed in reality.

In contrast, failure involving interfibre fracture takes place formally in an initially variable plane parallel to the fibres and is more complicated than FF. Figure 7a shows the fracture plane of a unidirectional (UD) reinforced composite body under an arbitrary three-dimensional stress state according to a fibre-adapted coordinate system.

In order to permit the characterization of the different forms and mechanisms of fracture by means of newgeneration failure criteria, it is advisable to introduce a natural coordinate system $x_{1}, x_{\mathrm{n}}, x_{\mathrm{t}}$. The orientation of the coordinate system is characterized by the rotation around the fibre-parallel $x_{1}$ axis (Fig. 7b). The fracture angle is directly incorporated in the failure criterion. Through the introduction of the rotatable system, not only the usual material stress can be predicted but also the fracture direction, which is of crucial importance for local damage analysis.

The corresponding criterion for IFF in general form is

$$
\max _{\theta} f\left(\sigma_{\mathrm{n}}, \tau_{\mathrm{nt}}, \tau_{\mathrm{n} 1}\right)=1
$$

This approach, through logical extension of Mohr's hypothesis, pursues the idea that the stresses in the fracture plane determine the fracture limit of a material. This failure hypothesis for interfibre fracture is of a more complex nature since it has to combine different forms of failure.

In 1992, Puck proposed an extended failure criterion for improved strength analysis with regard to interfibre failure of UD fibre reinforced composites. It is based on the following fracture hypothesis:

1. Tensile region $\left(\sigma_{\mathrm{n}} \geqslant 0\right)$. If a fibre-parallel fracture surface appears, the fracture is caused jointly by the transverse tensile stress $\sigma_{\mathrm{n}}$ and the two shear stresses $\tau_{\mathrm{n} 1}$

a)

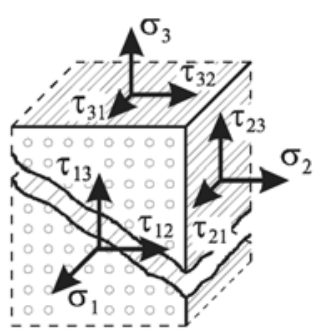

b)

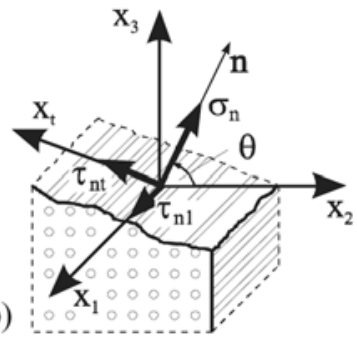

Fig. 7 UD basic element: (a) three-dimensional stress state in a fibre-adapted coordinate system; (b) stresses in the fracture plane

Proc. Instn Mech. Engrs Vol. 218 Part L: J. Materials: Design and Applications 
and $\tau_{\mathrm{nt}}$ acting in the fracture plane (at the moment of fracture)

$$
\left(\frac{\sigma_{\mathrm{n}}}{R_{\perp}^{(+)}}\right)^{2}+\left(\frac{\tau_{\mathrm{nt}}}{R_{\perp \perp}^{\mathrm{A}}}\right)^{2}+\left(\frac{\tau_{\mathrm{n} 1}}{R_{\perp \|}}\right)^{2}=1
$$

2. Compressive region $\left(\sigma_{\mathrm{n}}<0\right)$. A transverse compressive stress acting on the fracture plane makes no contribution towards initiating the fracture. On the contrary, it impedes the shear fracture caused by the shear stresses $\tau_{\mathrm{nt}}$ and $\tau_{\mathrm{n} 1}$ by giving rise to additional resistance to shear fracture; this resistance increases as a function of the compressive stress

$$
\left(\frac{\tau_{\mathrm{nt}}}{R_{\perp \perp}^{\mathrm{A}}-p_{\perp \perp} \sigma_{\mathrm{n}}}\right)^{2}+\left(\frac{\tau_{\mathrm{n} 1}}{R_{\perp \|}-p_{\perp \|} \sigma_{\mathrm{n}}}\right)^{2}=1
$$

The proportionality factors $p_{\perp \perp}$ and $\mathrm{p}_{\perp \|}$ in equation (19) cover the mentioned influence of the normal stress $\sigma_{\mathrm{n}}$ on failure in the compressive region owing to internal material friction. They can be obtained from experimental studies. The action plane resistance $R_{\perp \perp}^{\mathrm{A}}$ (A denotes the action plane), which occurs in both of the above fracture conditions in combination with the transverse/transverse shear stress $\tau_{\mathrm{n}}$, must not (as, for example, in the Hashin criterion) be replaced by the corresponding strength $R_{\perp \perp}$. The reason for this is that, unlike the tensile stress $\sigma_{\mathrm{n}}$ and the shear stress $\tau_{\mathrm{n} 1}$, the shear stress $\tau_{\mathrm{nt}}$ causes no failure of a UD laminate in its action plane.

A major advantage of the fracture condition with reference to an attached coordinate system is that, in particular, spatial stress states can be reliably evaluated with regard to failure. In contrast, the conventional generalized fracture criteria, especially in the case of three-dimensional stress conditions, often produce contradictory results, not least because they were developed and partly verified for plane, homogeneous fibre laminates. With the increasing applications of thick-walled fibre laminate components, however, it has also become necessary to consider the stresses perpendicular to the laminate plane, often critical for failure, in the design process [8]. The same applies to relatively thinwalled laminates of modern, highly anisotropic UD layers, because here critical stress combinations can also occur in planes parallel to the laminate plane (i.e. $\theta=90^{\circ}$ in Fig. $7 \mathrm{~b}$ ) owing to the strong deformation inhibitions.

\subsection{Failure analysis of conical composite rotors}

The main strain state of conical composite rotors is characterized by a coupling of primarily centrifugally induced stresses and secondarily induced bending stresses. This structural effect can easily be demonstrated in interaction diagrams based on reference [9]. For the assessment of these three-dimensional stress states, the Hashin/Puck failure criterion has been applied. As an example, the results of the stress analysis for thick-walled tangentially wound rotors

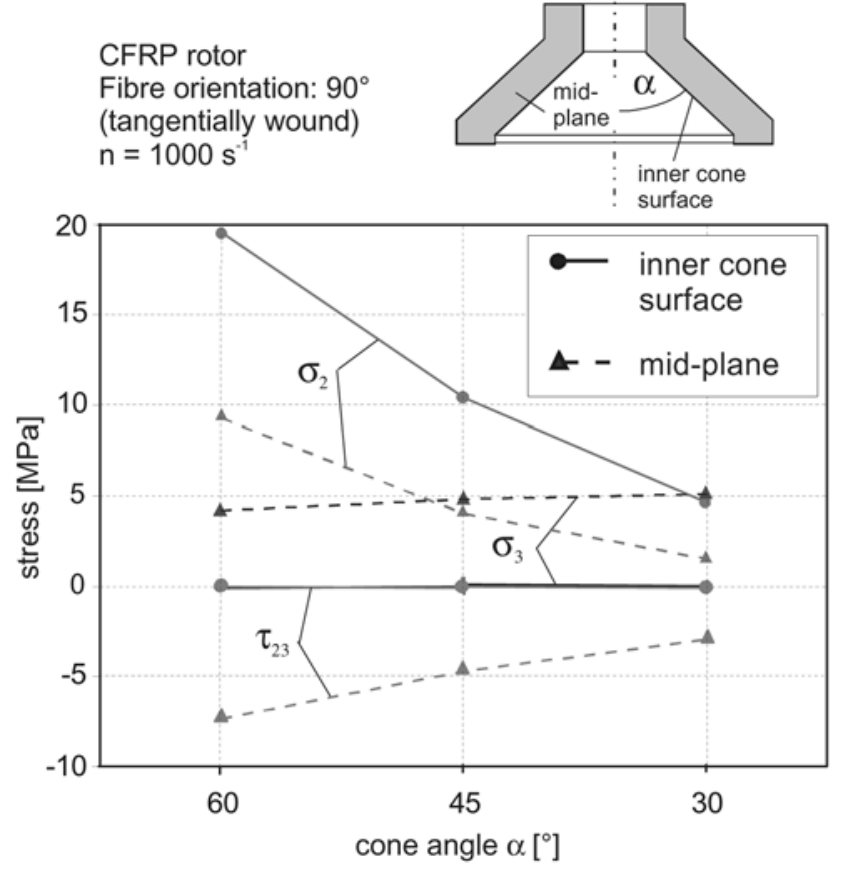

Fig. 8 Influence of cone angle on critical stresses

of conical shape with different cone angles $\alpha$ are shown in the interaction diagram in Fig. 8.

The stresses at the inner radius of the cone noticeably decrease for decreasing cone angles. This is of special interest for the failure critical stress $\sigma_{2}$, which acts perpendicular to the fibres. The reduction in the corresponding maximum stresses at the cone mid-plane is significantly lower for increasing cone angles. The stresses $\sigma_{3}$ of the mid-plane, however, increase for decreasing cone angles and, together with $\sigma_{2}, \sigma_{1}$ and $\tau_{23}$, result in a complex three-dimensional stress state as opposed to the plane stress state at the cone surface.

Owing to the change from a two-dimensional stress state at the cone surface to a three-dimensional stress state at the cone mid-plane, the greatest danger of fracture occurs for rotors with larger cone angles because of the increasing bending effects and the resulting tensile stresses perpendicular to the fibres (Fig. 9).

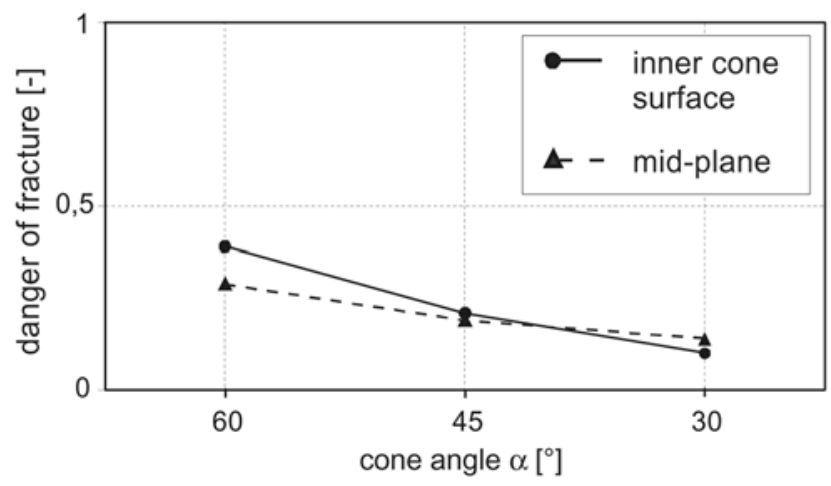

Fig. 9 Influence of cone angle on danger of fracture 


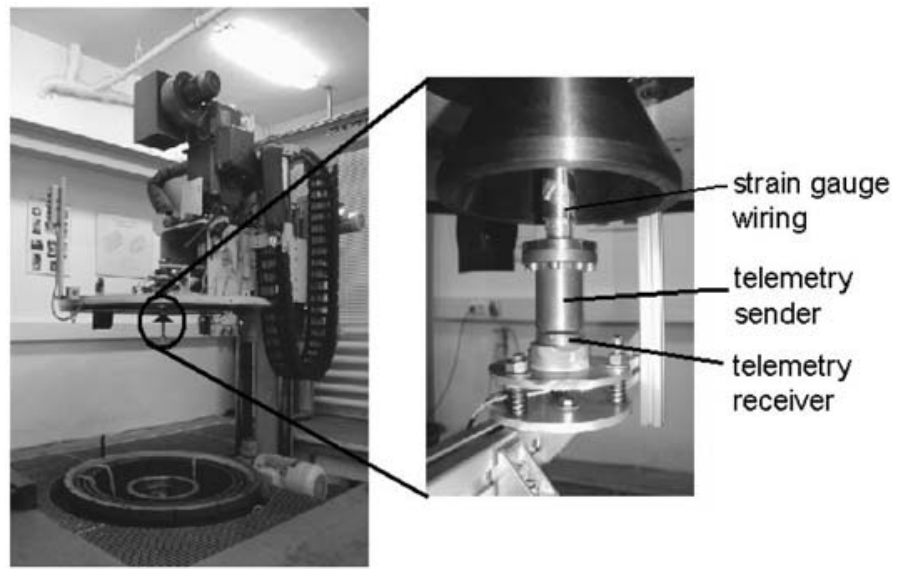

Fig. 10 Conical CFRP rotor with a telemetry system mounted in the spin unit

For the investigated conical rotors with higher cone angles, the bending effects and the resulting stresses at the inner cone surface are most critical, whereas for rotors with lower cone angles the three-dimensionally strained rotor mid-plane gains in importance with respect to failure owing to decreasing bending effects. Thus, for the design of thick-walled conical composite rotors, the stress states at the rotor surface as well as at the rotor mid-plane have to be analysed and assessed by physically based failure criteria dependent on the rotor shape.

\section{EXPERIMENTAL VERIFICATION}

For the experimental verification of the developed analytical methods and numerical models for non-cylindrical composite rotors under centrifugal forces and media as well as thermal loading conditions, multiple rotor tests of conical
CFRP rotors have been carried out using high-speed rotor testing units at the ILK. Maximum rotor speeds of $250000 \mathrm{r} /$ min in combination with elevated temperatures for rotor dimensions up to $1 \mathrm{~m}$ can be realized with these testing units (Fig. 10). Special telemetry-based on-line measurements of strain, temperature, etc., are used to verify the calculated structural behaviour of the wound rotor components.
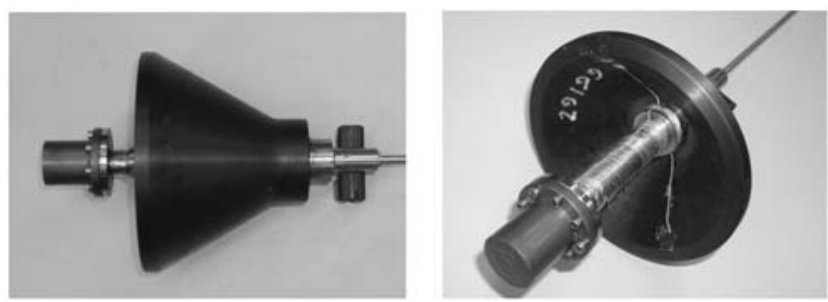

Fig. 11 Conical CFRP rotors with measurement equipment

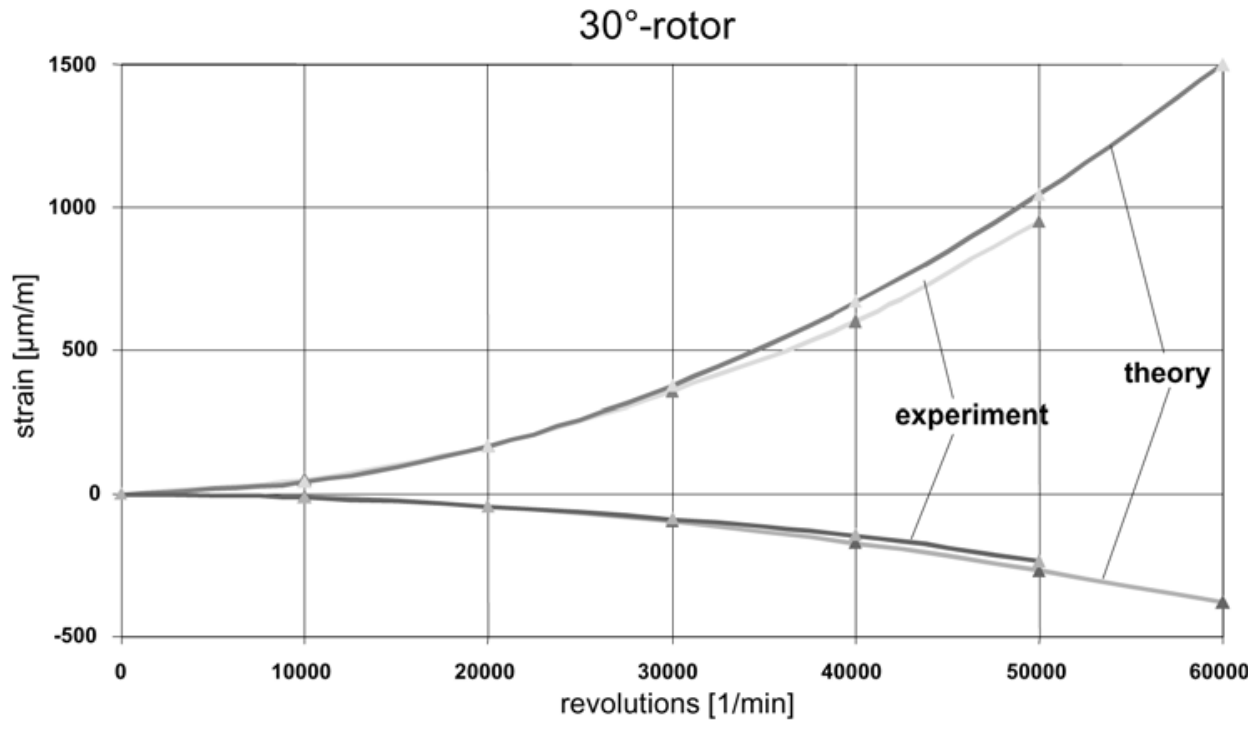

$\rightarrow$ phi, experiment $\rightarrow$ s, calculation $\quad--$ phi, calculation $\quad-$ s, experiment

Fig. 12 Theoretically and experimentally determined strain curves for a $30^{\circ}$ cone rotor 
For verification of theoretically obtained strain fields, conical CFRP rotors have been equipped for testing with a telemetry sender and strain gauges at the inner cone surface in the meridian and tangential directions (Fig. 11). A comparison of the experimentally obtained strains in the tangential and meridian directions and the corresponding theoretically determined strains is given in Fig. 12 for a $60^{\circ}$ cone rotor as an example, which shows very good agreement between the calculated and measured values.

Besides the quasi-static strain measurements, burst tests on cone-shaped rotors have been performed to verify the burst loading. During these experiments with composite rotors, effects that had not been known for metallic rotors have been observed. These burst effects result in loadings of the containment in the radial and tangential direction, which is known for metallic rotors, as well as in the axial direction, which is neglected especially for steel rotors, but has caused serious harm to the environment for composite rotors in the past [10].

\section{CONCLUSIONS}

In rotationally symmetrical but non-cylindrical rotors - such as conical rotors - additional bending effects due to centrifugal forces are superimposed on the known radial and tangential stress field from cylindrical and disc-shaped rotors. Consequently, the structural behaviour of noncylindrical rotors is characterized by a three-dimensional stress state. For the structural analysis of conical composite rotors, an analytical method has been developed that can advantageously be used in the design process and for extensive parameter studies with regard to the influence of multiple design variables of multilayered lightweight rotors such as cone angle, layer thickness and layer orientations.

The analytical method for three-dimensional analysis has successfully been verified by finite element calculations and numerous experimental high-speed rotor tests. Thus, besides the exact determination of three-dimensional stress states and critical stresses with regard to failure, the refined analytical calculation method is an efficient design tool for structural optimization, whereas optimization by the finite element method requires an extremely high modelling and computational effort.

\section{ACKNOWLEDGEMENT}

The financial support of the Arbeitsgemeinschaft industrieller Forschungsvereinigungen 'Otto von Guericke' e.V. (AiF) is gratefully acknowledged.

\section{REFERENCES}

1 Hufenbach, W., Kroll, L., Cedzich, R. and Grothaus, R. Ultra-light high-performance rotors for food processing technologies. Mechanika. Vol. 61, Wissenschaftliche Hefte der TU Oppeln, 2000, 256, 153-160.

2 Bogdanovich, A. E. and Pastore, C. M. Mechanics of Textile and Laminated Composites, 1996 (Chapman and Hall, London).

3 Grothaus, R., Hufenbach, W. and Kroll, L. Versagensanalyse und Bersteffekte bei faserverstärkten Rotor- und Containmentstrukturen. Verbundwerkstoffe und Werkstoffverbunde (Eds B. Wielage und G. Leonhardt), 2001, pp. 30-36 (Wiley$\mathrm{VCH}$, Weinheim).

4 Grothaus, R. and Hufenbach, W. Fracture and failure effect analysis of composite flywheel rotors and lightweight containment systems. 9th European Conference on Composite Materials (ECCM 9), Brighton, UK, 2000.

5 Kroll, L. and Hufenbach, W. Physically based failure criterion for dimensioning of thick-walled laminates. Appl. Composite Mater. 1997, 4, 321-332.

6 Hashin, Z. Failure criteria for unidirectional fibre composites. J. Appl. Mechanics, 1980, 47, 329-334.

7 Puck, A. Festigkeitsanalyse von Faser-Matrix-LaminatenModelle für die Praxis, 1995 (Carl Hanser Verlag, Munich).

8 Hufenbach, W., Kroll, L., Langkamp, A. and Gude, M. Realistische Festigkeitskriterien für Faserverbundwerkstoffe mit spröder Matrix. Verbundwerkstoffe und Werkstoffverbunde (Eds K. Schulte und K. U. Kainer), 1999, pp. 319-324 (Wiley-VCH, Weinheim).

9 Hufenbach, W., Kroll, L. and Grothaus, R. Interlaminares Versagen von Hybridrotoren, DECHEMA-Arbeitsausschuss 'Bauteilverhalten unter mechanischer Beanspruchung', Frankfurt, 14.11.96.

10 Hufenbach, W., Kroll, L. and Grothaus, R. Development, testing and containment design of advanced composite rotors. 19th International SAMPE Europe Conference, Paris, 1998, pp. $723-732$. 